\title{
Tissue plasminogen activator for acute ischemic stroke: Is the CAEP Position Statement too negative?
}

\author{
Jerome R. Hoffman, MD
}

A dapting information from research to clinical practice can be difficult, and we are often forced to decide whether benefits reported in selected trials are adequate to justify widespread application of the intervention studied. This is particularly problematic when the "evidence" is scanty, the degree of benefit apparently small (if real), and the treatment potentially dangerous. In addition, it is always important to consider whether community physicians can replicate the success described in research studies, which are typically performed by experts working in the controlled setting of a well-funded study.

To complicate matters, the use or non-use of new treatments may be subjected to the retrospective scrutiny of a courtroom whenever bad outcomes occur. Medicolegal concerns put even greater pressure on clinicians to adopt a new therapy, even if they are not convinced of a positive risk-benefit ratio. This is particularly true when external forces (e.g., advertising leading to public demand, review articles written by authors with financial ties to the manufacturer, and questionable guidelines created by "experts") speak in its favour.

The US Surgeon General's enthusiastic public endorsement of steroids for spinal cord injury, which was based on minimal and controversial evidence, led many physicians to accept high-dose steroids as the standard of care, despite widespread personal misgivings and published critiques. Similarly, recommendations by groups such as the American Heart Association (AHA) exert strong practical and legal weight on practitioners to use other disputed modalities or therapies with which they may not agree.

The Position Statement on thrombolytic therapy for acute ischemic stroke, recently published by the CAEP Committee on Thrombolytic Therapy for Acute Ischemic Stroke, ${ }^{1}$ addresses an important area where, despite ongoing debate and controversy, there is pressure on the emergency medicine (EM) community to consider this treatment a "standard of care" issue. This is in large part because of official support by organizations such as the AHA. ${ }^{2}$ In the US, more and more lawsuits are being filed against emergency physicians (EPs) for non-use of tPA, even if the decision to withhold it was based on an informed consideration of its relative risks and potential benefits, in the circumstances of both a given individual patient and a particular emergency department setting. This is reason enough for EPs to welcome the CAEP position paper, which is thoughtful and well-reasoned, and bases its conclusions on a careful evaluation of the available evidence. Although some may not agree with all of its recommendations, we should salute the courage of the authors and of CAEP in standing up to substantial outside pressure and producing such a reasonable document.

\section{The evidence}

The "evidence" in favour of tPA in acute stroke comes essentially from a single source. ${ }^{3}$ The well-designed but small National Institute of Neurologic Disorders and Stroke (NINDS) rt-PA Stroke Study, involving just 312 patients who received tPA, is the only randomized controlled trial (RCT) to show benefit from intravenous thrombolysis. Numerous other RCTs (some cited in the CAEP document) failed to show benefit and many were stopped early because of excessive mortality in treated patients. These conflicting results may reflect differences in study design, but could also reflect the play of chance.

When numerous studies of a therapy are performed, the study outcomes do not always represent truth. Rather, they produce a bell-shaped curve of results, with many approximating the "true" outcome, some being overly negative, and some being overly positive. Positive trials are more likely to be published and negative trials more likely to dis- 
appear (publication bias). There are numerous examples in medicine where a single study or a few small studies seemed to support a promising hypothesis, but subsequent larger work failed to confirm that benefit, or proved substantial harm. Ironically, one such example was published immediately after the NINDS trial in the same issue of the New England Journal of Medicine. The study ${ }^{4}$ found that low-molecular-weight heparin provided similar benefit to that reported for tPA in the NINDS trial. However, subsequent larger trials failed to confirm this benefit, and lowmolecular-weight heparin therapy for stroke has largely been abandoned.

\section{NINDS — Our landmark is flawed}

Even ignoring the many negative trials of stroke thrombolysis, there are further worries about accepting the NINDS results as "the truth." These relate to elements of the study design, the small number of stroke victims who qualify for lytic therapy (thus the limited potential for benefit), and the far larger number who stand to be harmed if this treatment, which is associated with substantial risk, is applied imperfectly.

An important methodologic concern about the NINDS trial is that it selectively enrolled patients with less than 90 minutes of symptoms. In fact, the study protocol required that investigators recruit equal numbers of "very early" patients (treated in 0-90 $\mathrm{min}$ ) and "early" patients (91-180 min). The study showed an overall $11 \%-13 \%$ absolute benefit with tPA treatment; however, a recent report by the NINDS authors ${ }^{5}$ clarified that the benefits were greater than this in the "very early" (0-90 min) group, which means they had to be less than this in the early (90-180 min) group. This is extremely important because, in real clinical practice, "very early" patients are almost non-existent. Had a disproportionate sampling mechanism not been built into the trial, it is likely that "very early" patients would have comprised a minimal proportion of those enrolled — and that the apparent benefit of treatment would diminish or even disappear. Thus, if "very early" patients were the group who derived most or all the benefit in NINDS, the only study to suggest benefit, evidence for the use of tPA becomes far more tenuous.

Another concern is that, in the 91-180 minute group, patients who received placebo were much sicker at baseline than those treated with tPA. Sicker patients tend to have worse outcomes, and these baseline differences may explain much of the apparent benefit that has been attributed to $\mathrm{tPA}$. These problems make it unclear whether there was any benefit to the use of tPA. If so, it is almost certain that the time limit for benefit is far less than 180 minutes, and perhaps much closer to 90 minutes.

\section{Efficacy vs. effectiveness}

Despite the above concerns, the CAEP Committee on Thrombolytic Therapy accepted the NINDS results at face value and did not object to the use of tPA in the hands of experts using the NINDS criteria. I am, however, far less sanguine about this, and I believe we should demand far better evidence of efficacy (benefit when used under optimal circumstances) before endorsing this treatment outside of ongoing research protocols. Far more important, the CAEP Position Statement appropriately challenges the effectiveness (benefit when used in ordinary practice) of tPA and suggests that most EPs should not apply this treatment outside of research or formal clinical protocols. I enthusiastically endorse this position, which I believe is strongly supported by the best information we currently have.

Only $1 \%-4 \%$ of stroke patients meet NINDS inclusion criteria, suggesting that, even accepting the NINDS numbers at face value, the potential benefit for the entire population of stroke patients is likely to be minimal. ${ }^{6,7}$ Given the risk associated with stroke thrombolysis and the likelihood that it will be misused at least some of the time by nonexperts, the effectiveness of tPA would almost certainly be marginal, at best. And with even a few errors, like treating "stroke mimics," failing to identify small intracranial hemorrhages on CT, or violating suggested eligibility criteria in a few patients, we could easily reach a situation where harm exceeds benefit. ${ }^{89}$ This concern is heightened by the fact that, unlike patients who actually have ischemic stroke, those with "stroke mimics" (e.g., hypoglycemia, post-ictal states) usually arrive early and "qualify" for thrombolytic therapy. Such patients - who cannot benefit from tPA but can be harmed by it - are also less likely to have other exclusion criteria, such as an abnormal CT scan or persistent hypertension.

The most compelling effectiveness study published to date comes from Cleveland..$^{10}$ Unlike a handful of other reports claiming to evaluate effectiveness, this paper did not involve sponsorship by the drug's manufacturers, nor experts who participated in the formal randomized clinical trials. It does not reflect selective reporting or publication bias, since it includes every single stroke patient treated at all 29 non-VA hospitals in the metropolitan Cleveland area over one year. In this study, only 70 (1.8\%) of 3948 patients hospitalized with acute ischemic stroke received tPA, and only half of these $(<1 \%)$ actually met NINDS eligibility criteria. The results in this real world sample are strik- 
ingly different from those reported in the NINDS trial, and from other selective "effectiveness studies" that had proprietary support. In fact, the Cleveland results were extremely and startlingly negative.

The $15.7 \%$ rate of symptomatic intracerebral hemorrhage was more than twice that in NINDS, and half of these were fatal. Predicted mortality in tPA treated patients was $7.9 \%$, but actual in-hospital mortality for patients treated with tPA was extremely high, at $15.7 \%$, compared with $7.2 \%$ for matched controls who presented within 3 hours of symptom onset but did not receive tPA. Interestingly, outcomes of patients meeting appropriate eligibility criteria were not notably different than outcomes of "protocol violators." Although the study lacked adequate power to prove that the outcomes in appropriate candidates were as bad as those in protocol violators, the outcomes were clearly terrible in both groups.

The Cleveland results were, of course, derived from a relatively small number of patients treated with tPA, and it was not a randomized, controlled trial. But the striking divergence between NINDS and the Cleveland experience reminds us of the large difference between efficacy in a research trial and effectiveness in real-world practice.

It is disturbing that the AHA upgraded its classification of early tPA for stroke to "Class I" based on only one positive RCT, especially as the decision was made at about the same time that the Cleveland results were published in JAMA. It is hard to imagine how these results, which raise sobering doubts about whether NINDS can carry over into widespread practice, did not give the AHA more pause. Add in the questions about NINDS itself, and the recent reevaluation of its results, ${ }^{5}$ and the AHA decision seems startling indeed.

It is worth emphasizing that other "effectiveness" studies that support the community use of tPA provide much less compelling evidence. A good example is the CASES database (www.strokeconsortium.ca/CASES). This is an Internet site, supported by a manufacturer of tPA, which invites physicians to register cases and provide unsubstantiated "outcomes" of treatment. The voluntary reporting is, of course, likely to be extremely selective, and the "outcomes" not only depend on scoring systems that are subject to substantial interpretation (and thus possible bias), but which also require no verification. These weaknesses makes this "database" so far from science that I wonder why anyone would use it to justify a treatment that has a striking potential to harm patients.

\section{Summary}

It is clear that no consensus exists regarding the appropri- ateness of tPA for acute ischemic stroke. The CAEP committee's willingness to acknowledge this, to recognize the limitations of the therapy, and to recommend appropriate caution, is a tremendously positive step. It should be applauded heartily by all of us who do our best to make difficult decisions, under enormous time stresses - regardless of whether we suspect that tPA will ultimately prove, on balance, and in community practice, to do more good, or more harm, in patients with suspected stroke.

\section{References}

1. Canadian Association of Emergency Physicians Committee on Thrombolytic Therapy for Acute Ischemic Stroke. Thrombolytic therapy for acute ischemic stroke. CJEM 2001;3(1):8-12.

2. American Heart Association. Guidelines 2000 for cardiopulmonary resuscitation and emergency cardiovascular care. Circulation 2000;102(Suppl I). Available: http://circ.ahajournals .org/content/vol102/suppl_1/\#ECC_GUIDELINES (accessed 2001 June 02).

3. Tissue Plasminogen Activator for Acute Ischemic Stroke. The National Institute of Neurological Disorders and Stroke rt-PA Stroke Study Group. N Engl J Med 1995;333(24):1581-7.

4. Kay R, Wong KS, Yu YL, Chan YW, Tsoi TH, Ahuja AT, et al. Low-molecular-weight heparin for the treatment of acute ischemic stroke. N Engl J Med 1995;333(24):1588-93

5. Marler JR, Tilley BC, Lu M, Brott TG, Lyden PC, Grotta JC, et al. Early stroke treatment associated with better outcome: the NINDS rt-PA stroke study. Neurology 2000;55:1649-55.

6. Jorgensen HS, Nakayama H, Kammersgaard LP, Raaschou HO, Olsen TS. Predicted impact of intravenous thrombolysis on prognosis of general population of stroke patients: simulation model. BMJ 1999;319:288-9.

7. Hoffman JR. Predicted impact of intravenous thrombolysis. Another trial is needed [letter]. BMJ 2000;320:1007.

8. Libman RB, Wirkowski E, Alvir J, Rao TH. Conditions that mimic stroke in the emergency department: implications for acute stroke trials. Arch Neurol 1995;52(11):1119-22.

9. Schriger DL, Kalafut M, Starkman S, Krueger M, Saver JL. Cranial computed tomography interpretation in acute stroke: physician accuracy in determining eligibility for thrombolytic therapy. JAMA 1998;279:1293-7.

10. Katzan IL, Furlan AJ, Lloyd LE, Frank JI, Harper DL, Hinchey JA, et al. Use of tissue-type plasminogen activator for acute ischemic stroke: the Cleveland area experience. JAMA 2000; 283(19):1151-8.

Correspondence to: Dr. Jerome R. Hoffman, UCLA Emergency Medicine Center, 924 Westwood Blvd., Ste. 300, Los Angeles CA 90077; jrh@ucla.edu 